\title{
Varicoid Carcinoma of the Esophagus
}

\author{
Terry M. Silver, MD, and Harvey M. Goldstein, MD
}

The radiologic diagnosis of esophageal carcinoma ordinarily is not difficult. Infrequently, however, a polypoidal, infiltrative form of squamous cell carcinoma may closely resemble varices (1-3). Careful attention to the fluoroscopic and roentgenographic appearance is essential for correct diagnosis.

\section{CASE REPORTS}

\section{Case 1}

A 63-year-old Oriental male was evaluated for symptoms of a cough and a choking sensation of 1 year's duration. There was no history of either weight loss or dysphagia. Past medical history and physical examination were noncontributory.

A barium swallow revealed several thickened, irregular, longitudinal filling defects in the middle and lower esophagus. The distal $4 \mathrm{~cm}$ were normal (Figure 1A). There was no variation in the size or shape of the defects from film to film, utilizing deep inspiration and the Müller and Valsalva maneuvers. At fluoroscopy, the diseased portion of the esophagus was not distensible and peristaltic activity was absent.

Endoscopy and subsequent surgery confirmed extensive neoplastic involvement with distinct ulcerated ridges of tumor. Histologic diagnosis was well-differentiated squamous cell carcinoma with invasion of all esophageal layers and periesophageal structures. The patient expired in the early postoperative period from diffuse bilateral pneumonia.

\section{Case 2}

A 59-year-old male was admitted with a 4-month history of progressive dysphagia and weight loss, and a past history of pulmonary tuberculosis. The patient admitted to intermittent heavy drinking, but there was no history of liver disease or jaundice. Emaciation was the salient physical finding.

From the Department of Radiology, University of Michigan Medical Center, Wayne County General Hospital, Eloise, Michigan.

Address for reprint requests: Terry M. Silver, MD, Department of Radiology, University Hospital, Ann Arbor, MI 48104.
Fluoroscopic and radiographic examination of the esophagus demonstrated multiple, irregular, haphazard filling defects in the lower two-thirds of the esophagus (Figure 1B). Some narrowing of the esophagus at the upper and lower extent of the lesion was present. No change in esophageal contour was noted with repeated swallows of barium.

At esophagoscopy there were multiple friable masses projecting into the lumen. Biopsy revealed squamous cell carcinoma. Radiotherapy was initiated but the patient expired with progressive inanition in 1 month, despite a feeding gastrostomy.

\section{DISCUSSION}

The irregular, intraluminal filling defects of varicoid carcinoma may appear identical to true esophageal varices on any given radiograph (Figure 1C). The distinction must be made at fluoroscopy and/or with a series of films. With varices, the esophagus remains distensible and has peristaltic activity. In addition, on serial radiographs, varicosities will change in size and shape, particularly during the Müller and Valsalva maneuvers. This is in contrast to the rigidity and lack of peristalsis in cases of infiltrative, polypoidal carcinoma. Predictably, no change in the appearance of the filling defects occurs with respiratory maneuvers in carcinoma. An abrupt demarcation between the abnormal area and adjacent normal esophageal segment is also a helpful differential point favoring neoplasm (3).

The lack of involvement of the distal esophagus was another useful finding in one of our cases (Figure 1A). Varices resulting from superior vena caval obstruction also typically spare the distal esophagus, but changeability will be noted, as it is with varices due to portal hypertension (4).

Esophagoscopy will usually confirm the diagnosis of neoplasm if friable ulcerated intraluminal masses are seen. However, longitudinal

Digestive Diseases, Vol. 19, No. 1 (January 1974) 

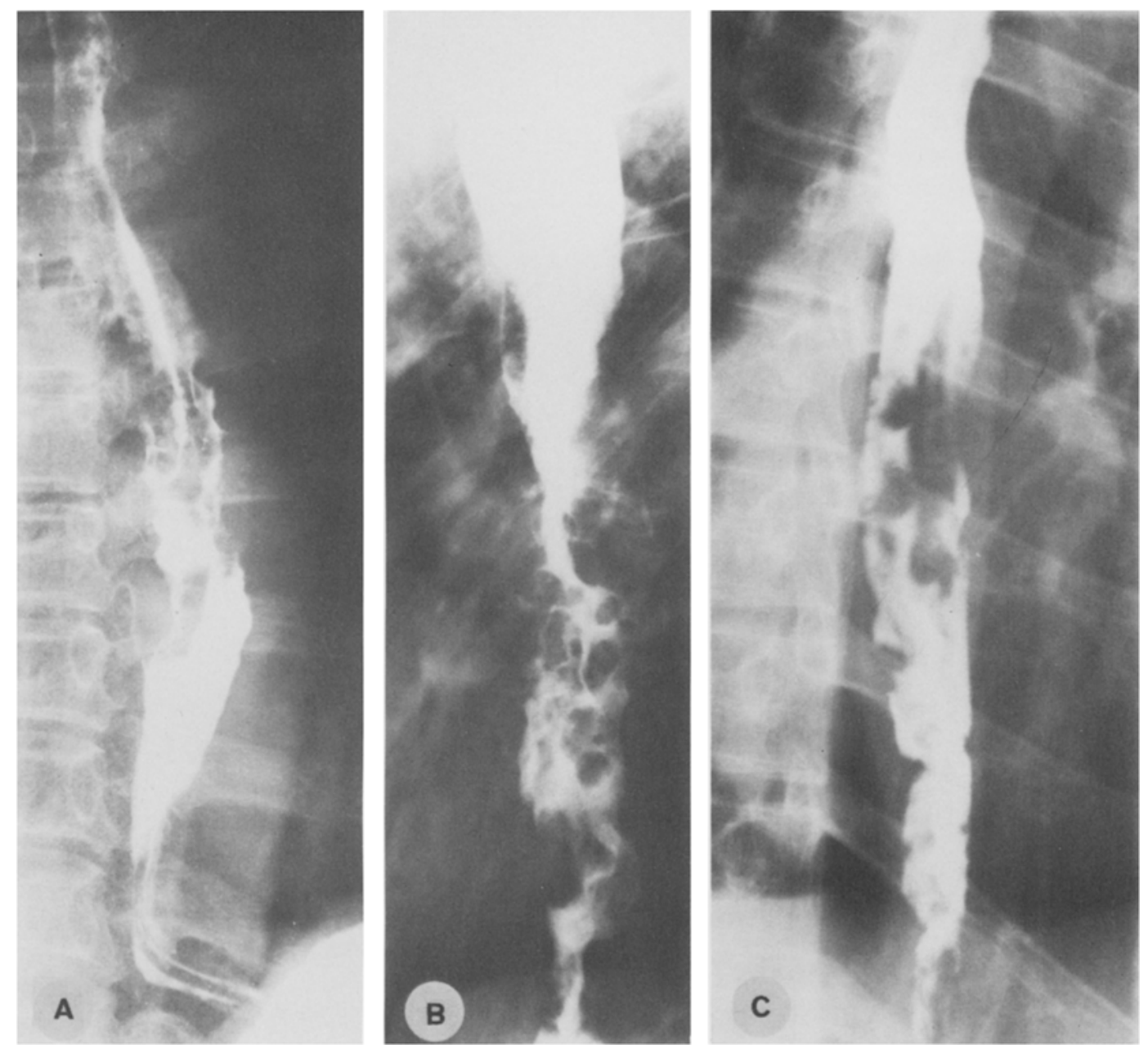

Fig 1A. Case 1. Carcinoma of the esophagus. Frontal view of the esophagus demonstrates varicoid filling defects with sparing of the distal esophagus. There was no change in their appearance on serial films, and at fluoroscopy peristalsis in the diseased segment was absent. Fig 1B. Case 2. Carcinoma of the esophagus. Lateral view of the esophagus shows multiple, irregular, polypoidal defects resembling varices. At fluoroscopy, no change in esophageal contour was observed. Fig 1C. Esophageal varices. Barium swallow demonstrates longitudinal and ovoid filling defects which changed in size and shape at fluoroscopy. On a single radiograph, the close similarity between varices and varicoid carcinoma is striking.

tumor ridges have been confused with varices in at least one recorded instance (2).

Since esophageal obstruction is a late manifestation of varicoid carcinoma, the relative lack of presenting symptoms may be misleading. As a result, considerable intramural and extramural neoplastic infiltration may occur before obstructing symptoms with dysphagia are present.

\section{SUMMARY}

Two cases of esophageal squamous cell carcinoma closely resembling esophageal varices on radiographic examination have been presented. Rigidity and absence of peristalsis at 
fluoroscopy, as well as no change in appearance of the polypoidal filling defects with spot filming, differentiate varicoid carcinoma from esophageal varices. The lack of involvement of the distal esophagus was helpful in differentiating carcinoma from varices in one of the cases.

\section{REFERENCES}

1. Brombart M: Clinical Radiology of the Esopha- gus. Baltimore, Williams \& Wilkins Co, 1961, p 339

2. Lawson TL, Dodds WJ, Sheft DJ: Carcinoma of the esophagus simulating varices. Am J Roentgen $107: 83-85,1969$

3. Prévôt R: Die Röntgendiagnostik der ösophagusvarizen als frühdiagnose der pfortaderstauung. Roentgenpraxis 12:85-90, 1940

4. Felson B, Lessure AP: "Downhill" varices of the esophagus. Dis Chest 46:740-746, 1964. 